\section{Identification of a miR-146b-Fas ligand axis in the development of neutropenia in $T$ large granular lymphocyte leukemia}

Barbara Mariotti, ${ }^{1 *}$ Giulia Calabretto ${ }^{2,3 *}$ Marzia Rossato, ${ }^{1 \S}$ Antonella Teramo Monica Castellucci, ${ }^{1 \dagger}$ Gregorio Barilà, ${ }^{2,3}$ Matteo Leoncin, ${ }^{2,3}$ Cristina Vicenzetto, ${ }^{2,3}$ Monica Facco, ${ }^{2,3}$ Gianpietro Semenzato, ${ }^{2,3}$ Flavia Bazzoni, ${ }^{1, \star}$ and Renato Zambello $2,3, *$

${ }^{1}$ Department of Medicine, Division of General Pathology, University of Verona, Verona; ${ }^{2}$ Department of Medicine, Hematology and Clinical Immunology section, University of Padua, Padua and ${ }^{3}$ Venetian Institute of Molecular Medicine (VIMM), Padua, Italy

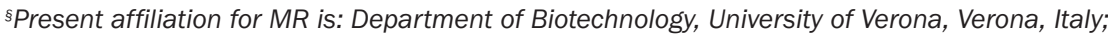

${ }^{+}$Present affiliation for MC is: Genomics and transcriptomics platform, CPT, University of Verona, Verona, Italy

${ }^{\star} B M, G C, F B$ and $R Z$ contributed equally to this work.

\section{ABSTRACT}

large granular lymphocyte leukemia (T-LGLL) is characterized by the expansion of several large granular lymphocyte clones, among which a subset of large granular lymphocytes showing constitutively activated STAT3, a specific CD8 ${ }^{+} / \mathrm{CD}^{-}$- phenotype and the presence of neutropenia has been identified. Although STAT3 is an inducer of transcription of a large number of oncogenes, so far its relationship with miRNAs has not been evaluated in T-LGLL patients. Here, we investigated whether STAT3 could carry out its pathogenetic role in T-LGLL through an altered expression of miRNAs. The expression level of 756 mature miRNA was assessed on purified $\mathrm{T}$ large granular lymphocytes (T-LGLs) by using a TaqMan Human microRNA Array. Hierarchical Clustering Analysis of miRNA array data shows that the global miRNome clusters with CD8 T-LGLs. Remarkably, CD8 T-LGLs exhibit a selective and STAT3-dependent repression of miR$146 \mathrm{~b}$ expression, that significantly correlated with the absolute neutrophil counts and inversely correlated with the expression of Fas ligand (FasL), that is regarded as the most relevant factor in the pathogenesis of neutropenia. Experimental evidence demonstrates that the STAT3-dependent reduction of miR-146b expression in CD8 T-LGLs occurs as a consequence of miR$146 \mathrm{~b}$ promoter hypermethylation and results in the disruption of the HuRmediated post-transcriptional machinery controlling FasL mRNA stabilization. Restoring miR-146b expression in CD8 T-LGLs lead to a reduction of HuR protein and, in turn, of FasL mRNA expression, thus providing mechanistic insights for the existence of a STAT3-miR146b-FasL axis and neutropenia in T-LGLL.

\section{Introduction}

T large granular lymphocytes leukemia (T-LGLL) is a rare disease characterized by the abnormal expansion of $T$ large granular lymphocytes (T-LGLs) in the peripheral blood. ${ }^{1,2}$ While the aetiology of the disease is still elusive, LGL proliferation is thought to be maintained through an impairment of the apoptotic machinery due to the activation of many survival signals. ${ }^{3}$ Among these, JAK/STAT signalling represents one of the most important deregulated pathways in T-LGLL. In particular, leukemic LGLs are equipped with STAT3 constitutively over-expressed and in some cases over-activated. ${ }^{4,5}$ Moreover, in $30-40 \%$ of patients, STAT3 has been demonstrated to carry hot-spot mutations, likely resulting in STAT3 activation. ${ }^{6,7}$ This genetic lesion was also correlated by some authors with increased frequency of neutropenia $a^{5,7,8}$ which represents the most frequent cause of symptomatic dis-
Ferrata Storti Foundation

Haematologica 2020

Volume 105(5):1351-1360

\section{Correspondence:}

FLAVIA BAZZONI

flavia.bazzoni@univr.it

Received: May 3, 2019.

Accepted: August 23, 2019.

Pre-published: August 29, 2019.

doi:10.3324/haematol.2019.225060

Check the online version for the most updated information on this article, online supplements, and information on authorship \& disclosures: www.haematologica.org/content/105/5/1351

\section{(C)2020 Ferrata Storti Foundation}

Material published in Haematologica is covered by copyright. All rights are reserved to the Ferrata Storti Foundation. Use of published material is allowed under the following terms and conditions:

https://creativecommons.org/licenses/by-nc/4.0/legalcode. Copies of published material are allowed for personal or internal use. Sharing published material for non-commercial purposes is subject to the following conditions:

https://creativecommons. org/licenses/by-nc/4.0/legalcode, sect. 3. Reproducing and sharing published material for commercial purposes is not allowed without permission in writing from the publisher. 
ease in LGLL patients, often requiring specific therapy. The pathogenesis of neutropenia, although not properly elucidated thus far, seems to be multifactorial. Multiple mechanisms have been postulated, including destruction of mature neutrophils and myeloid progenitors via Fas/Fas ligand (FasL). Mechanisms leading to neutropenia are not completely characterized, although $\mathrm{we}^{5}$ and others ${ }^{9-12}$ provided evidence that extremely high concentrations of FasL are detectable in T-LGLL patients suggesting that soluble FasL is involved in this process. We also demonstrated that STAT3 plays a central role in FasL transcription and we showed that high levels of STAT3 activation correlated with high levels of FasL expression in T-LGLL patients. ${ }^{5}$ However, the molecular mechanism through which STAT3 regulates FasL production in these patients has not yet been clarified.

Although STAT3 is an inducer of transcription of a large number of oncogenes, its relationship with microRNAs (miRNAs) has not yet been evaluated in T-LGLL patients. As a matter of fact, several miRNAs contribute to normal hematopoietic processes and many miRNAs act both as tumor suppressors and oncogenes in the pathogenesis of hematological malignancies, including acute and chronic leukemias and lymphomas, where they contribute to lymphomagenesis acting in various cellular functions, such as the regulation of cell survival and proliferation. . $^{13,14}$

In this study a high throughput quantitative and qualitative analysis of the miRNA expression profile in leukemic LGLs was performed with the aim to investigate the role of miRNAs in clinical and biological features of T-LGLL, thus playing a role in the pathogenesis of neutropenia in T-LGLL patients.

\section{Methods}

\section{Patients}

Thirty T-LGLL patients and nine age and sex-matched healthy donors were enrolled in the study. Clinical and biological characteristics of patients are summarized in the Online Supplementary Table S1. At the time of the study, no patients had received treatment, with a follow-up ranging from 1 to 16 years. LGL proliferation ranged from $48 \%$ to $91 \%$ of the lymphocyte pool. T-LGLL clonality was demonstrated as previously reported. ${ }^{15}$ The Padova Institutional Review Board approved this study and written informed consent in accordance with the Declaration of Helsinki was obtained by each subject.

\section{Cell isolation and culture}

T-LGLs were purified from peripheral blood mononuclear cells (PBMC) using the anti-CD57 microbeads (Milteny Biotec), as previously reported. ${ }^{16} \mathrm{CD} 8{ }^{+} \mathrm{CD} 57^{+}$cells used as control, were obtained from PBMCs of healthy donors by the FACSAria cell sorter (BD biosciences). Purity and viability of cell preparation were both $>95 \%$. The LGL phenotype was assessed by flow cytometry. In selected experiments PBMC from T-LGLL patients $\left(2 \times 10^{6}\right.$ cells $\left./ \mathrm{mL}\right)$ were cultured in RPMI1640 (EuroClone) supplemented with 10\% FCS (Sigma-Aldrich), $2 \mathrm{mM}$ Gln $25 \mathrm{mM}$ Hepes, $100 \mathrm{U} / \mathrm{mL}$ penicillin and $100 \mu \mathrm{g} / \mathrm{mL}$ streptomycin (EuroClone) in the presence of $2.5 \mu \mathrm{M}$ 5-aza-2'-deoxycytidine (DAC, SigmaAldrich) for three days, $15 \mu \mathrm{M}$ STATTIC (Shelleckchem) for 24 hours (h) or DMSO (Sigma-Aldrich) as control.

\section{miRNA and Gene-Expression Analysis}

RNA was purified from CD57+ cells using the miRCURY RNA
Isolation Kit (Exiqon) according to the manufacturer's instructions. High throughput and single miRNA expression were analysed using the TaqMan ${ }^{\circledR}$ Human microRNA Array (Card Set v3.0, Applied Biosystems) and the TaqMan ${ }^{\circledR}$ microRNA Human Assays (Applied Biosystems), respectively, as previously described. ${ }^{17}$ Expression of DNMT1, FasL, FasL-Primary Transcript (PT), HuR, GAPDH, pri-miR-146b and RPL32 mRNA was quantified by realtime quantitative PCR (RT-qPCR) as previously described, ${ }^{18}$ using specific primer pairs listed in the Online Supplementary Table S2.

\section{Methylated DNA Immunoprecipitation (meDIP) assay}

Genomic DNA was purified from $\mathrm{CD} 57^{+}$T-LGLs with the AllPrep DNA/RNA/miRNA Universal Kit (Qiagen), according to the manufacturer's instructions and methylated DNA Immunoprecipitation (meDIP) assay was performed as reported by Mohn et al. ${ }^{19}$ with minor modifications. The methylation levels of miR-146b promoter was analysed by qPCR using specific primers (Invitrogen, Thermo Scientific) listed in the Online Supplementary Table S2.

\section{Western blot}

T-LGLs $\left(2.5 \times 10^{5}\right)$ and Jurkat cells were lysed in Sample Buffer (40mM tris (hydroxymethyl) aminomethane $\mathrm{HCl} \mathrm{pH} 6.8,7.5 \%$ glycerol, $1 \%$ sodium dodecyl sulphate). Total cell lysates were resolved on SDS-PAGE and transferred onto nitrocellulose (Millipore). The blots were incubated with specific antibodies and detected using the ImageQuant LAS 500 or the Odyssey infrared imaging system (LI-COR Biosciences).

\section{Cell transfection}

Jurkat cells $\left(10^{6}\right)$ or freshly purified CD57 ${ }^{+}$T-LGL $\left(10^{7}\right)$ were transfected with the indicated amount of specific oligonucleotide using the Amaxa Nucleofector (Lonza) and the Ingenio Electroporation Solution (Mirus Bio).

\section{Enzyme-linked immunosorbent assay (ELISA)}

Secreted FasL in plasma were determined by ELISA kit (RayBiotech, Georgia, USA), following the manufacturer's recommendations.

\section{Statistics}

Statistical evaluation was performed by using the MannWhitney U-test, the Wilcoxon signed-rank test or Student $t$-test, as appropriate, with $\alpha$ set to 0.05 . Correlation coefficient was determined using the Spearman Rank Correlation.

For detailed information on the materials and methods used, see the Online Supplementary Material and Methods.

\section{Results}

\section{Characterization of T-LGL miRNome}

To identify miRNAs potentially involved in T-LGLL, the miRNA pattern of expression was investigated in CD57+ cells purified from six patients affected by T-LGLL and three healthy donors, using Taq-Man Human microRNA Array. Unsupervised Hierarchical Clustering Analysis (HCA) of normalized array data led to the identification of two clusters: cluster A including LGL1, LGL3, LGL5 and LGL9, and cluster B including LGL10, LGL17 together with the three healthy donors (HD1, HD2, HD4), (Figure 1A). Interestingly, according to the characterization of the T-LGLL patients enrolled in the study, LGL samples in cluster A share the $\mathrm{CD} 4^{-} \mathrm{CD} 8^{+} \mathrm{CD} 16^{+} \mathrm{CD} 56^{-} \mathrm{CD} 57^{+}(\mathrm{CD} 8$ T-LGLs) immunophenotype, while LGLs in cluster B share 
A

Cluster A Cluster B

morn

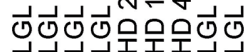
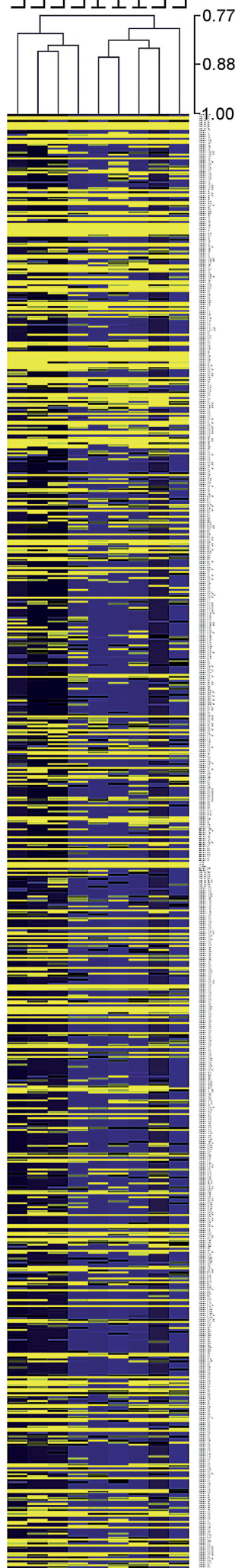

(2)

25

miRNA

expression (a.u.)
B
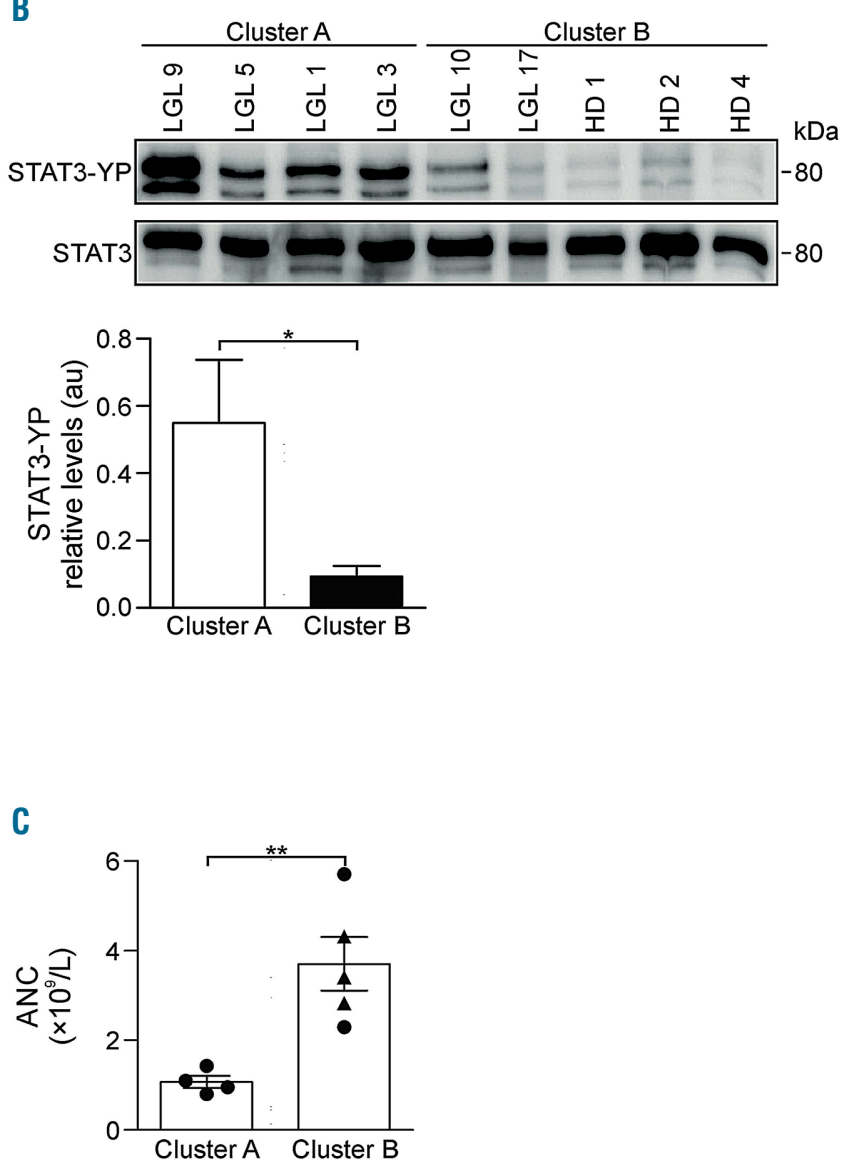

Figure 1. miRNome of T-LGLL patients correlates with STAT3 phosphorylation. Peripheral blood $\mathrm{CD} 57^{+} \mathrm{T}$ cells from $\mathrm{T}$ large granular lymphocyte leukemia (T-LGLL) patients $(n=6)$ and healthy donors (HD, n=3) were collected. (A) Heatmap representation of the 756 miRNAs analyzed using the TaqMan microRNA array as described in the Methods. Results are expressed as arbitrary units (au) using $\mathrm{U} 6$ as reference control. (B) Whole-cell extracts (250x10 cells) purified from the same cells as in (A) were loaded on gels and analyzed for STAT3-YP and total STAT3 expression level. Quantification of the relative normalized STAT3-YP protein levels are reported as arbitrary units (au) below the Western blot. Mean \pm standard error of the mean (SEM) is shown, $* P<0.05$ by unpaired $t$-test. (C) Absolute neutrophil counts in each CD8 and CD4 T-LGLL patient (circle) and HD (triangle). Mean \pm SEM is shown. $* * P<0.01$ by unpaired t-test. Patients analyzed in each panel are specified in the Online Supplementary Table S5. 
the $\mathrm{CD}_{4}{ }^{+} \mathrm{CD} 8^{+} \mathrm{CD} 16{ }^{-} \mathrm{CD} 56^{+} \mathrm{CD} 57^{+}$(CD4 T-LGLs) immunophenotype (Online Supplementary Table S1). We have recently shown that $\mathrm{CD} 8^{+} \mathrm{CD} 16^{+} \mathrm{CD} 56^{-} \mathrm{CD} 57 \pm$ immunophenotype identifies a subset of patients characterized by the presence of STAT3 mutation and/or activation and neutropenia, whereas STAT3 mutations are lacking in CD4 T-LGLL patients, usually displaying normal neutrophil counts. ${ }^{5}$ Confirming our previous observation, high levels of STAT3-YP, detected by Western blot analysis (Figure 1B), characterized the CD8 T-LGLL patients, three of whom carry STAT3 mutations (Online Supplementary Table S1). Conversely, no STAT3 mutations (Online Supplementary Table S1) nor constitutive activation (Figure 1B) were detected in CD4 T-LGLs. No variation in the total STAT3 levels between CD8 and CD4 samples was detected (Figure 1B). Moreover, and consistent with our previous observation, CD8 T-LGLL patients are characterized by a significantly $(P=0.0066)$ different absolute neutrophil counts (ANC) as compared to CD4 T-LGLL patients, that shows normal neutrophil counts (Figure 1C). Taken together, our data show that the global miRNome clusters with STAT3-activated/CD8 phenotype, that is, in turn, characterized by neutropenia.

\section{miR-146b is differentially expressed in CD8 versus CD4 T-LGLs and inversely correlates with neutropenia}

On the basis of the above correlation between the global mature miRNA expression profile and the presence of activated STAT3 (Figure 1), and taking into account that a pathogenic link between CD8 phenotype and STAT3 activation has been demonstrated, ${ }^{5}$ we hypothesized that a STAT3 activation-dependent, CD8-specific miRNAs expression pattern is in place. To get insights into this immunophenotype-specific miRNome, miRNAs expressed in CD8 and CD4 T-LGLs were subjected to differential expression analysis. miRNAs showing threshold cycle $(\mathrm{C} t$ ) value $<32$, and Fold Change (FC) value $>2$ or $<0.5$ were considered as differentially modulated. Accordingly, twenty-four miRNA emerged as up-regulated and only one miRNA, namely miR-146b, as down-regulated in a statistically significant manner $(P<0.05)$ in CD8 as compared to CD4 T-LGLs (Online Supplementary Figure S1 and Online Supplementary Table S3).

Based on our recent data indicating that high level of STAT3 activation correlates not only with CD8 T-LGLs phenotype, but also with the presence of symptomatic disease, mostly as a consequence of neutropenia, ${ }^{5}$ all the miRNAs differentially expressed in CD8 and CD4 T-LGL (Online Supplementary Table S3) were analysed for correlation with the ANC. Correlation analysis highlighted only two miRNAs, namely miR-630 and miR-146b, whose expression correlated with ANC $(P=-0.886, P=0.033$ and $P=0.866, P=0.030$, respectively) and simultaneously with the levels of STAT3-YP $(P=1.00, P=0.003$ and $P=-0.866$, $P=0.033$, respectively) (Online Supplementary Table S4). None of the remaining differentially modulated miRNA correlated with the absolute neutrophil count in a statistically significant manner (Online Supplementary Table S4). RT-qPCR single assay on additional T-LGLL patients confirmed the downregulation of miR-146b expression in CD8 T-LGLs, compared to CD4 T-LGLs $(P=0.018)$ or to T lymphocytes from healthy donors $(P=0.024)$ (Figure $2 \mathrm{~A})$. Accordingly, miR-146b was found to inversely correlate simultaneously with the levels of activated STAT3 $(P=-$ 0.846, $P=0.0005)$ and with ANC $(P=0.707, P=0.0012)$
(Figure 2B). Consistently, in the additional T-LGLL patients a significant correlation between STAT3 activation and neutropenia was confirmed as well $(P=-0.867$, $P=0.0003$ ) (Figure $2 \mathrm{~B}$, right panel). On the contrary, miR630 was confirmed as differentially expressed in CD4 T-LGLs as compared to CD8 T-LGLs, but the correlation with ANC was not validated (not shown).

To gain insights into the cause-effect relationship between the degree of STAT3 activation and the lack of miR-146b expression, CD8 T-LGLs were incubated with non-toxic doses of the STAT3 inhibitor STATTIC and the level of miR-146b expression was analyzed. Figure 2C shows that blocking STAT3 activity in CD8 T-LGLs unleashed miR-146b transcription, thus demonstrating that suppression of miR-146b expression in CD8 T-LGLs is secondary to constitutive STAT3 activation.

STAT3 is a well-known transcriptional activator for many genes ${ }^{20}$ and it has also been reported to inhibit gene expression by promoting methylation of the target genes promoter. ${ }^{21-23}$ In normal tissues STAT3 is reported to activate miR-146b transcription. ${ }^{24,25}$ However, in several systems miR-146b promoter methylation has been shown to prevent miR-146b expression, even in the presence of constitutively activated STAT3 ${ }^{26,27}$ According to the publically available methylome data (https://genomeeuro.ucsc.edu/cgi-bin/hgTracks?db=hg19 \&last VirtModeType=defaultellastVirtModeExtraState $=$ EvirtMode Type $=$ default\&virtMode $=0$ \&nonVirtPosition $=$ \&position $=$ chr1 o\%3A104196181104196428\&hgsid=2306888991_Xz5zjxAj b58tIT50L9i5MkaweCLP), four cytosine located upstream (-570bp, -63bp, -56bp, -26bp) and two located downstream (-71bp, and -273bp) the miR-146b TSS (Online Supplementary Figure S2) have been identified as differentially methylated in different cell lines. On these bases, we analyzed the level of miR-146b promoter methylation in CD8 and CD4 T-LGLs that are characterized by the presence or absence of activated STAT3, respectively. Our results showed a significantly higher level of $5 \mathrm{meC}$ in the regions $-687 \mathrm{bp} /-496 \mathrm{bp}(+141.21 \%, P<0.01)$ and $149 \mathrm{bp} /+98 \mathrm{bp}(+58.46 \%, P<0.05)$ upstream miR-146b TSS in CD8 T-LGLs compared to CD4 T-LGLs (Figure 3A). As a control, methylation of the region downstream $(+44 \mathrm{bp} /+315 \mathrm{bp}) \mathrm{miR}-146 \mathrm{~b}$ TSS in CD8 and CD4 T-LGL was comparable (Figure 3A). Inhibition of methyl-transferase activity with 5-aza-2-deoxycytidine (DAC) restored the expression of the miR-146b primary transcript (pri-miR-146b) in CD8 T-LGLs (Figure 3B), further proving that miR-146b promoter methylation prevents miR-146b expression in CD8 T-LGLs. Remarkably, STAT3 inhibition led to a statistically significant reduction of the level of DNMT1 expression, thus suggesting that constitutive STAT3 activation may lead to miR-146b promoter methylation (Figure 3B, left panel). Collectively, these data suggest a cause-effect link between the lack of miR-146b expression and constitutive STAT3-activation in CD8 T-LGLs.

\section{FasL mRNA-stabilizing protein HuR is target of miR-146b}

To gain insights into the molecular mechanisms underlying the correlation between miR-146b expression and ANC (Figure 2B, central panel), we focused our subsequent analysis on FasL. In fact, increased release of FasL has long been reported as one of the most relevant factor in the pathogenesis of neutropenia in LGLL patients.,12,28 
According to our published data, ${ }^{5}$ RT-qPCR analysis showed that FasL mRNA expression correlates with the degree of STAT3 activation $(P=0.762, P=0.0368$ ) (Figure $4 \mathrm{~A})$ and simultaneously inversely correlates with ANC $(P=-0.727, P=0.0096)$ (Figure 4B). Most importantly, an inverse correlation between miR-146b and FasL mRNA expression (Figure 4C) and the release of soluble FasL (sFasL) (Figure 4D) were detected. Consistently, FasL mRNA expression was higher in CD8 T-LGLL patients (MNE 0.0306 \pm 0.0067 ) compared to the non-neutropenic patients belonging to the CD4 T-LGLL subset (MNE $0.0119 \pm 0.0026, P=0.02$ ) (Figure 4E). Interestingly, no difference in the level of FasL primary transcript (FasL-PT) expression between CD8 and CD4 T-LGLs was observed (Figure 4E), suggesting that a mechanism controlling FasL expression at a post-transcriptional level is defective in CD8 T-LGLL subset, that therefore display higher levels of FasL mRNA as compared to CD4 T-LGLL subset.s
In order to demonstrate that miR146b and FasL expression were causally linked, miR-146b was overexpressed in Jurkat and the level of FasL mRNA was analyzed $48 \mathrm{~h}$ post transfection. miR-146b-overexpressing cells have reduced levels of FasL mRNA (MNE $2 \times 10^{-5} \pm 5.77 \times 10^{-6}$ ) compared to cells transfected with a scramble control miRNA (MNE $3.67 \times 10^{-5} \pm 6.67 \times 10^{-6}$ ) (Figure $5 \mathrm{~A}$ ), suggesting a role of miR$146 \mathrm{~b}$ in the regulation of FasL expression. Nevertheless, in silico miR-target prediction analysis performed by seven different target prediction software (microT4, miRanda, Pictar2, PITA, RNA22, miRWalk and TargetScan) did not identify FasL among the putative miR-146b target genes, thus suggesting that miR-146b eventually affects FasL expression indirectly, by targeting genes involved in FasL mRNA stability. Among the sixteen genes retrieved as putative miR-146b targets commonly predicted by all software only one, namely the ribonucleoprotein Human Antigen R (HuR, also known as ELAVL1), plays a well-

A

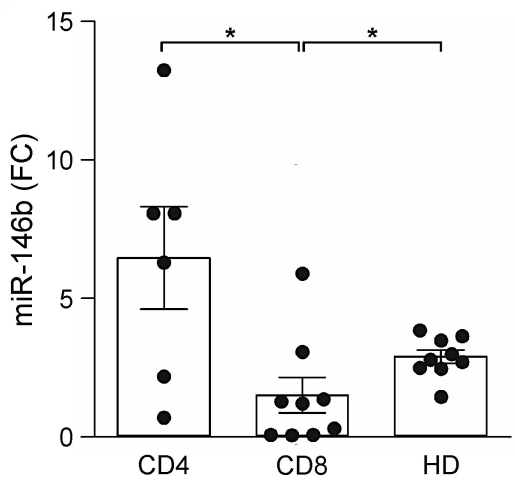

B
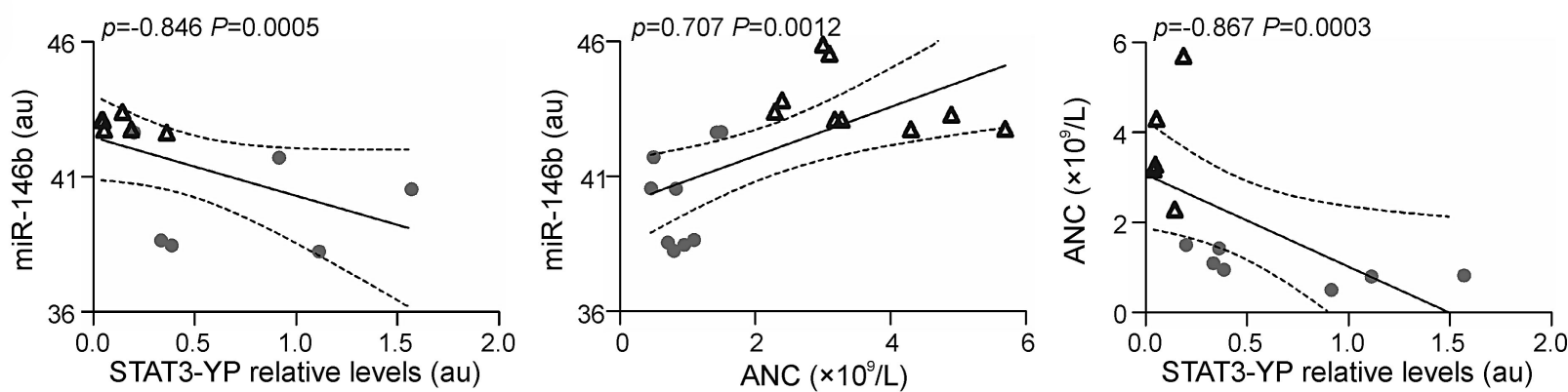

C

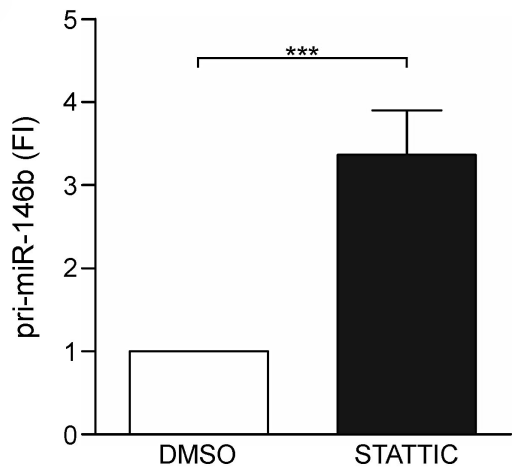

Figure 2. STAT3-dependent inhibition of $m i R-146 \mathrm{~b}$ expression in CD8 T large granular lymphocytes. (A) miR-146b expression level in each CD8 $(n=9)$ and CD4 $(n=6)$ T large granular lymphocytes (T-LGLs) as well as in HD $(n=9)$ together with mean+standard error of the mean (SEM) are shown. $* P<0.05$ by Kruskal-Wallis tests. (B) Correlation analysis between miR-146b expression and STAT3YP (left, $n=12$ ), miR-146b expression and absolute neutrophil count (ANC) (central, $n=18$ ), and between ANC and STAT3-YP levels (right, $n=12$ ). Grey circles identify CD8 T-LGLs, empty triangles identify CD4 T-LGLs. miR-146b expression analyzed in real-time quatitative PCR (RT-qPCR) is reported as arbitrary units after U6 normalization. The relative STAT3-YP protein levels, normalized for total STAT3, are reported as arbitrary units (au). ANC is reported as cell $\times 10^{\circ} / \mathrm{L}$. Spearman correlation coefficient ( $p$ ) and P-values are reported. (C) CD8 T-LGLs were cultured for 24 hours (h) in presence of $15 \mu \mathrm{M}$ STATTIC or DMSO as control $(\mathrm{n}=7)$. miR-146b primary transcript (pri-miR-146b) expression, analyzed by RT-qPCR, is reported as Fold Induction (FI) relative to DMSO treated cells, after RPL32 normalization. Mean \pm SEM are shown. $* * * P<0.001$ by Wilcoxon signed-rank. Patients analyzed in each panel are specified in the Online Supplementary Table S5. 
A
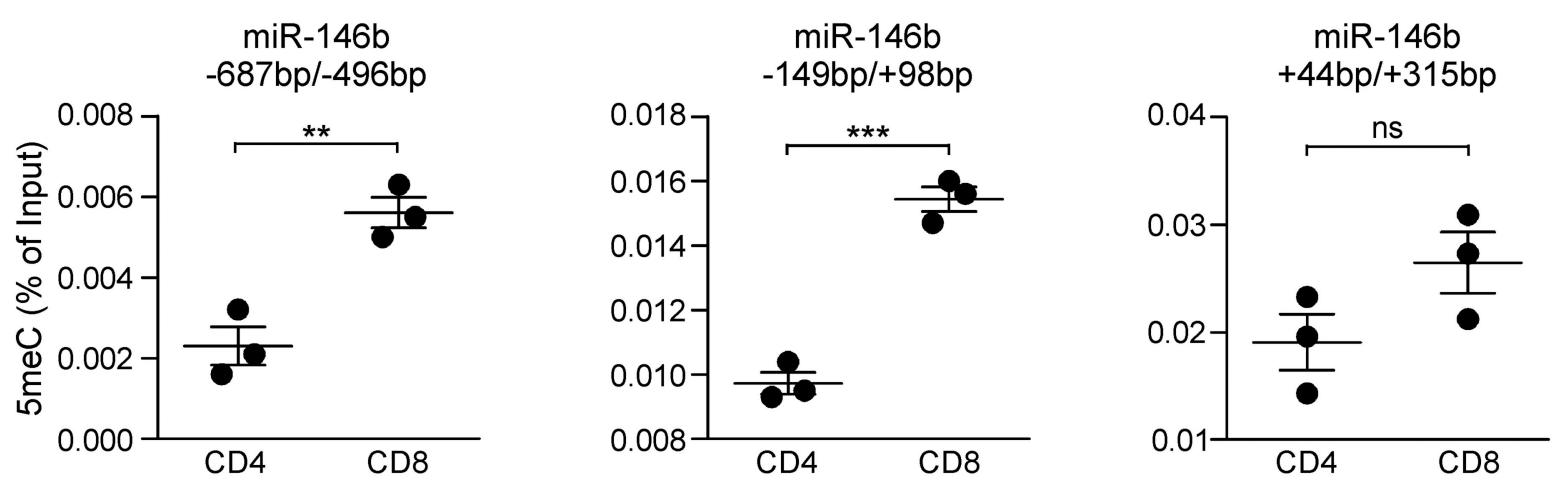

B
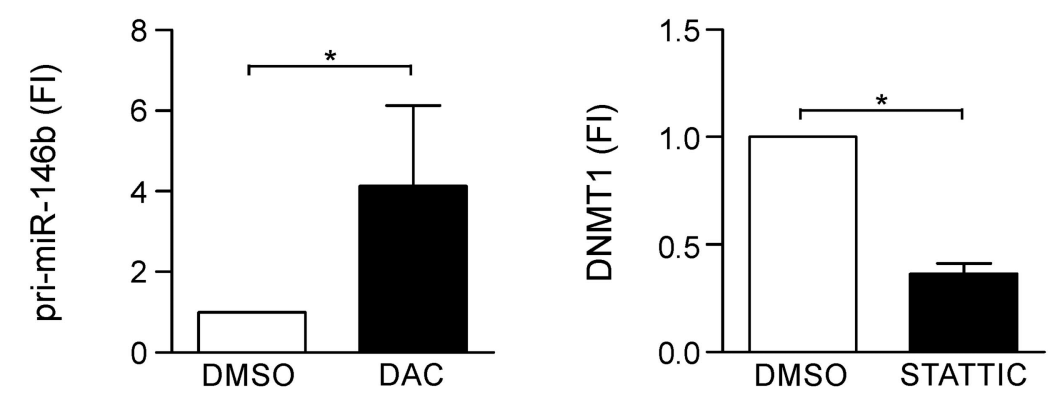

Figure 3. Promoter methylation prevents miR-146b expression in CD8 T large granular lymphocytes. (A) The methylation level of $-687 /-496$, $-149 /+98$ miR-146b promoter region and $+44 /+315$ downstream region was analyzed by meDIP assay with anti-5meC antibody (Ab) in CD4 ( $n=3)$ and CD8 ( $n=3$ ) T large granular lymphocyte leukemia (T-LGLS). Single value and mean \pm standard error of the mean (SEM) are reported as percentage over input. $* * P<0.01, * * * P<0.001$, not significant (ns) $P>0.05$ by unpaired t-test. (B) PBMCs from CD8 T-LGLL patients were cultured in presence of 2.5 uM 5-aza-2'-deoxycytidine (DAC) for three days (left) or $15 \mu \mathrm{M}$ STATTIC for 24 hours (h) (right). pri-miR-146b (left) and DNMT1 (right) expression was analyzed. Data are expressed as Fold Induction (FI) relative to the untreated condition (DMSO). Mean \pm SEM of six (left panel) and seven (right panel) independent experiments is shown. $* P<0.05$, by Wilcoxon signed-rank test. Patients analyzed in each panel are specified in the Online Supplementary Table S5.

defined role in mRNA stabilization, and has been reported to be absolutely required for FasL expression in T lymphocytes. ${ }^{29}$ Moreover, the potential miR-146b binding site in the 3' UTR of HuR (Online Supplementary Figure S3) has been demonstrated to be functional by 3'UTR reporter assay. ${ }^{30,31}$ Consistently, overexpression of miR-146b in Jurkat cells led to a reduction of the intracellular HuR protein levels $(-34.05 \pm 3.74 \%, n=2)$ (Figure $5 \mathrm{~B})$. Likewise, silencing HuR with a specific HuR siRNA caused a parallel reduction of FasL mRNA expression (Figure 5C), thus providing further evidence for the causal link between miR-146b-mediated reduction of the FasL mRNA-stabilizing protein $\mathrm{HuR}$ and the consequent decrease of FasL mRNA. Most importantly, restoring miR-146b expression in purified CD8 $\mathrm{T}$ cells from LGLL patients caused a reduction of $\mathrm{HuR}$ mRNA expression (Figure 6A), thus validating $\mathrm{HuR}$ as endogenous miR-146b target in primary CD8 T-LGLs as well. Concurrently, FasL mRNA, but not FasL primary transcript, expression was reduced (Figure $6 \mathrm{~A}$ ), thus demonstrating that miR-146b-mediated reduction of $\mathrm{HuR}$ protein post-transcriptionally controls FasL expression. To ascertain that HuR protein expression inversely correlates with the levels of endogenous miR$146 \mathrm{~b}$ expression, the intracellular levels of HuR protein in CD8 and CD4T-LGLs were examined. Remarkably, endogenous HuR protein was detected in CD8 T-LGLs, that express low levels of miR-146b, at levels significantly higher $(P=0.003)$ than those detected in miR-146bexpressing CD4 T-LGLs (Figure 6B). Collectively, these data show that in CD8 T-LGLs constitutively activated STAT3 leads to the loss of miR-146b, that in turn unleashes the translation of HuR protein. As a consequence, HuR stabilizes FasL mRNA and increases FasL production, causing, in turn, neutropenia.

\section{Discussion}

MicroRNAs are regarded as important gene expression regulators often involved in the pathogenesis of a variety of conditions such as cancer and autoimmunity. In this study, by exploring a relevant number of miRNAs, we report the first characterization of a restricted pattern of miRNAs differentially expressed in T-LGLL patients. Unsupervised hierarchical clustering analysis identified a correlation between the miRNA expression profile and individual T-LGL subset, characterized by a specific CD8 ${ }^{+}$ phenotype, by high levels of constitutive STAT3 phosphorylation and by the presence of specific clinical features. Noticeably, miRNA expression correlates mostly with STAT3 activation status, rather than with the presence of STAT3 mutations. Several authors suggested a pathogenic 
A

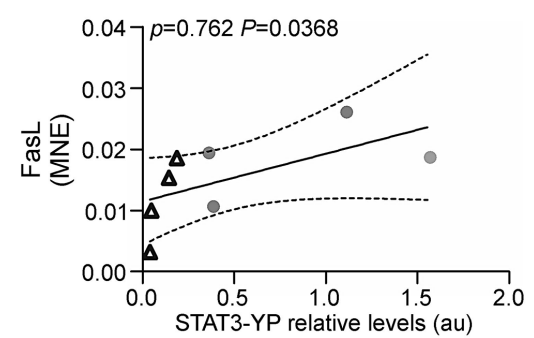

D

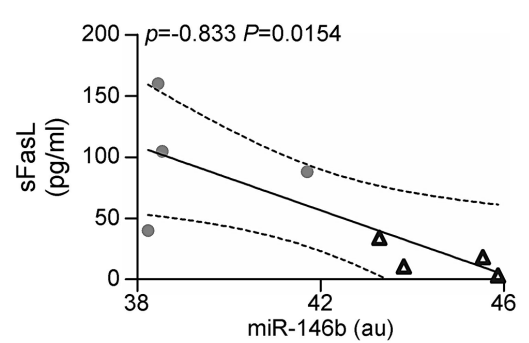

B

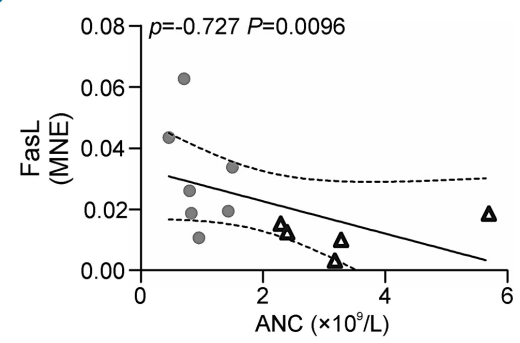

E

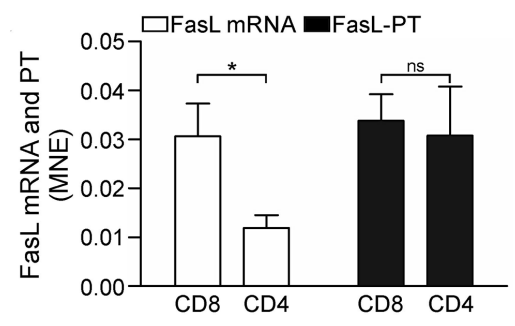

C

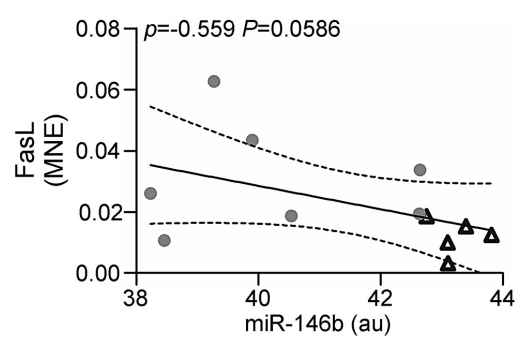

Figure 4. miR-146b controls Fas ligand mRNA expression targeting HuR. Correlation analysis between Fas ligand (FasL) mRNA expression and STAT3-YP levels (A; $n=8)$, FasL mRNA expression and ANC (B; $n=12)$, FasL mRNA expression and miR-146b expression levels $(C ; n=12)$ and soluble FasL (sFasL) and miR-146b expression levels ( $D ; n=8)$. FasL mRNA is expressed as MNE relative to GAPDH, while miR-146b is expressed as au after $U 6$ normalization. ANC is reported as cellx10 $/ \mathrm{L}$ and sFasL is expressed in $\mathrm{pg} / \mathrm{mL}$. Spearman correlation coefficient $(p)$ and $P$ are reported. (E) FasL mRNA and FasL primary transcript (PT) were analyzed in CD8 $(n=7)$ and CD4 $(n=5)$ T-LGLs by real-time quatitative PCR (RT-qPCR). Data are expressed as MNE relative to GAPDH. Data are reported as mean \pm standard error of the mean (SEM). ${ }^{\star} P<0.05$, ns, not significant by Mann-Whitney U-test. Grey circles identify CD8 T-LGLs, empty triangles identify CD4 T-LGLs. Patients analyzed in each panel are specified in the Online Supplementary Table S5.

A

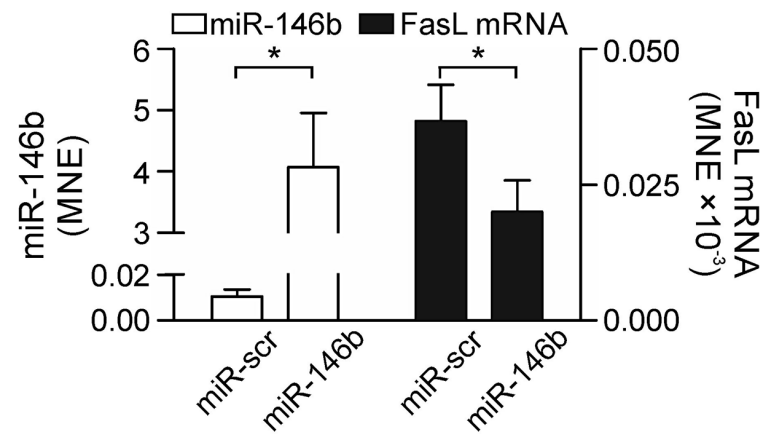

B
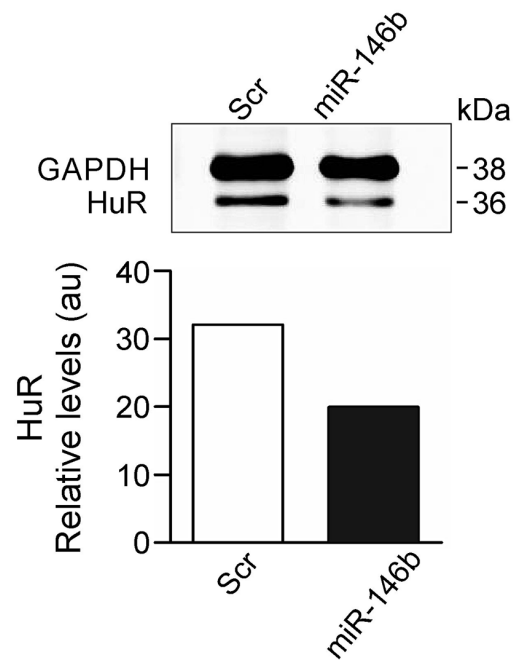

GAPDH

HuR
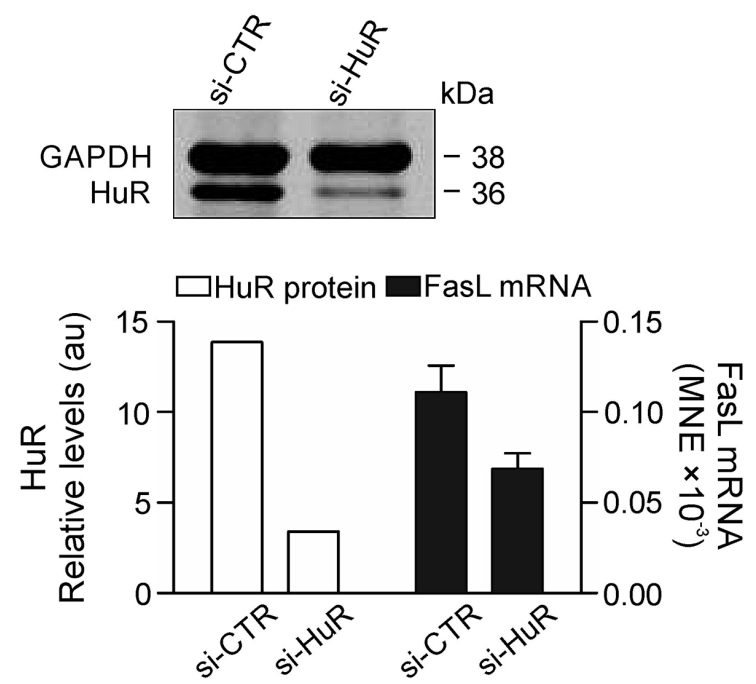

Figure 5. miR-146b regulates Fas ligand expression by targeting HuR in Jurkat cells. (A) Jurkat cells were transfected with $75 \mathrm{pmol}$ miR-scr or miR-146b. 48 hours (h) after transfection cells were processed and miR-146b and FasL mRNA expression were analyzed. Data are expressed as Mean Normalized Expression (MNE) relative to U6 (miR-146b) and GAPDH (FasL mRNA). Mean \pm standard error of the mean (SEM) $(n=3)$ is shown. ${ }^{*} P<0.05$ by paired $t$-test. (B) Jurkat cells were transfected with $75 \mathrm{pmol}$ miR-scr or miR-146b. $48 \mathrm{~h}$ after transfection cells were processed and HuR protein level was analyzed by western blotting. One Western blot representative of two performed with similar results is shown. The relative HuR protein level, normalized for GAPDH, is reported as au below the Western blot. (C) Jurkat cells were transfected with 200 pmol si-CTR or si-HuR and processed as described above. One Western blot representative of two performed with similar results is shown. Quantification of normalized HuR protein level and of FasL mRNA expression (Mean \pm SEM, $n=2$ ) in transfected Jurkat is shown below the Western blot. 
A

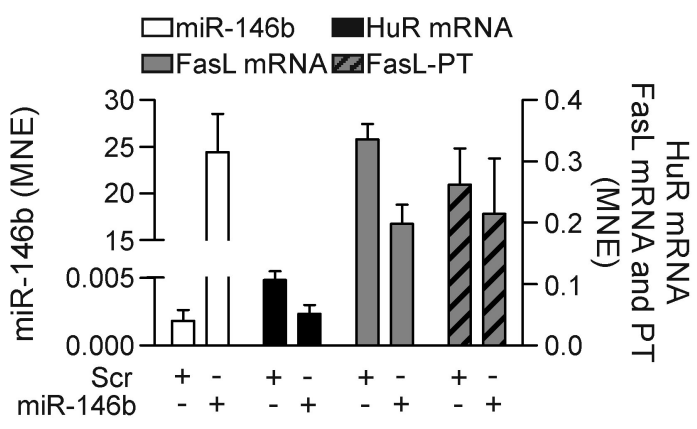

B
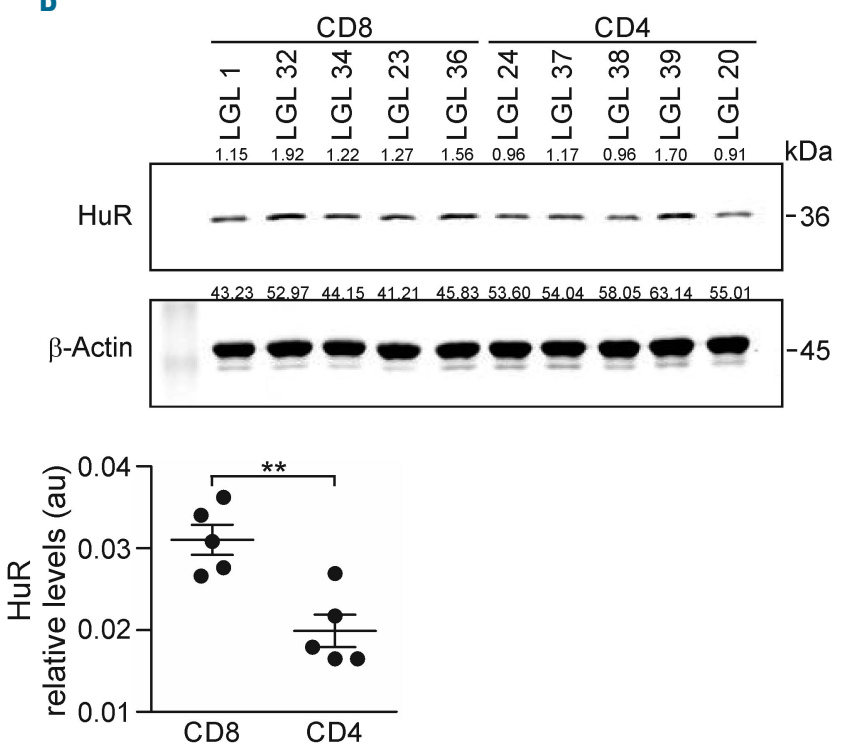

Figure 6. HuR is the endogenous miR-146b target gene in CD8 T large granular lymphocytes. (A) CD8 T large granular lymphocytes (T-LGL) were transfected with 200 pmol miR-scr or miR-146b. 24 hours (h) after transfection cells were processed and miR-146b, HuR mRNA, FasL mRNA and primary transcript (PT) expression were analyzed by real-time quatitative PCR (RT-qPCR). Data are expressed as MNE relative to U6 (miR-146b) and RPL32 (HuR, FasL mRNA and PT). Mean \pm standard error of the mean (SEM) of two independent experiments is shown. (B) HuR protein level was analyzed by Western blot as described in the Methods in CD8 ( $\mathrm{n}=5$ ) and CD4 $(n=5)$ whole-cell extracts $(15 \mu \mathrm{g})$. Normalized HuR protein levels are reported as arbitrary units (au) below the Western blot. * $P<0.01$ by unpaired $t$-test. Patients analyzed in each panel are specified in the Online Supplementary Table S5.

role for STAT3 mutations in this disease. ${ }^{6,7}$ However these mutations, mostly of the activating type, involve a variable percentage of pathological clones and in many cases are present in a very low percentage of LGL. ${ }^{32}$ Our results point to the role of STAT3 activation as the dominant factor in the pathogenesis of the disease and in the induction of a specific miRNA profile. These findings are consistent with recent data from our lab indicating a correlation between STAT3 activation, phenotypic pattern of proliferating LGLs and the presence of symptomatic disease, mostly characterized by neutropenia. ${ }^{5}$

The mechanism sustaining neutropenia in LGLL patients still remains poorly clarified. Since infiltration of pathological LGLs usually play only a marginal role in the pathogenesis of neutropenia, soluble factors have been reported to be the more relevant players in this feature. Among them, FasL has been detected at very high concentrations in LGLL patients. ${ }^{9,12,28}$ In particular, a significant increase in FasL mRNA and protein expression was reported in patients with $\mathrm{CD} 8^{+} \mathrm{CD} 16^{+} \mathrm{CD} 56^{-}$phenotype. ${ }^{5,9-}$ ${ }^{12}$ Consistently, we also found that the CD8 T-LGL population under investigation is characterized by higher levels of FasL expression. Comparative analysis of the differentially expressed miRNAs within different T-LGL subsets allowed us to identify miR-146b as a unique miRNA. In fact, miR-146b expression is decreased in CD8 T-LGLL, that is distinguished from the CD4 T-LGLL phenotype by high levels of constitutive STAT3 activation. Moreover, miR-146b expression inversely correlates with the levels of STAT3 tyrosine phosphorylation, with neutropenia and concurrently with FasL expression, suggesting the existence of a STAT3-miR146b-FasL axis in T-LGL leukemia. Inhibition of constitutively activated STAT3 by STATTIC in CD8 T-LGLL patients increased miR-146b expression, thus providing experimental evidence for a mechanistic link between constitutively activated STAT3 and inhibition of miR-146b expression in CD8 T-LGLs. This finding is in line with data showing that induction of miR-146b expression by STAT3 occurs under normal physiological conditions only in non-transformed cells, but is lost in malignancy, mostly has a consequence miR-146b promoter methylation, that prevents miR-146b expression even in the presence of constitutively activated STAT3 ${ }^{26,27}$ Here we provide the first evidence of $\mathrm{miR}-146 \mathrm{~b}$ promoter methylation at the expected sites, thus pointing that a similar mechanism might also take place in CD8 T-LGLL. Inhibition of this process using DAC restored miR146b levels. Moreover, a direct role of activated STAT3 in inducing $\mathrm{miR}-146 \mathrm{~b}$ promoter methylation, through regulating expression of DNA methyltransferase 1 (DNMT1) has been demonstrated in solid tumors ${ }^{27}$ and in malignant T lymphocytes. ${ }^{33}$ Similarly, we show that inhibition of constitutively active STAT3-YP reduces the expression of DNMT1, thereby providing a functional and mechanistic link between activation of STAT3 signaling pathway and its epigenetic control. Interestingly, a role for epigenetic mechanisms taking place in chronic LGL proliferations has been already reported by Caligiuri et al. in T-LGLL ${ }^{34}$ and by our group in CLPD-NK, ${ }^{35}$ and our data contribute to unravel the machineries differently activated in each subsets of patients (i.e. $\mathrm{CD}^{+}$vs. $\mathrm{CD}^{+} \mathrm{LGLL}$ ) with relevant clinical impact.

Restoration of miR-146b expression in Jurkat cells and, most importantly, in patients CD8 T-LGLL, resulted in a significant reduction of the FasL mRNA expression level, which occurs in the absence of modification of the FasL primary transcript expression. Collectively, these data indicate that miR-146b affects FasL expression at a post- 

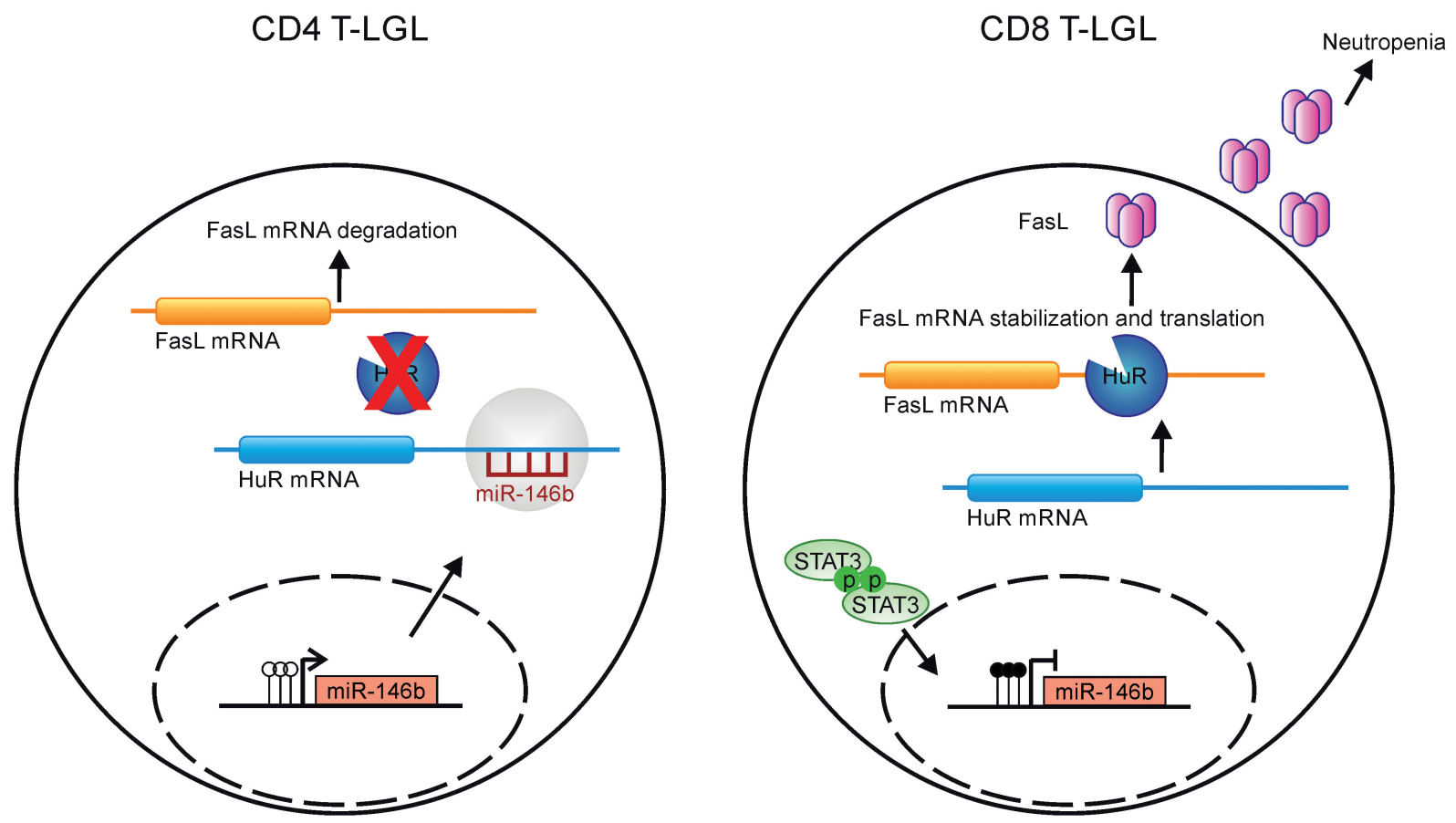

Figure 7. Schematic representation of the pathogenic link between constitutively active STAT3, defective miR-146 $b$ expression and Fas ligand-mediated neutropenia, that specifically characterizes the CD8 subset of large granular lymphocyte leukemia. Methylation status of miR-146b promoter is represented as circles: empty circles mean not methylated cytosine, while black circles represent methylated cytosine.

transcriptional level. Nevertheless, in silico miR-target prediction analysis did not identify FasL among the putative miR-146b target genes, thus suggesting that miR-146b eventually affects FasL expression indirectly, by targeting genes involved in FasL mRNA stability. In fact, we demonstrated that the intracellular mRNA level of HuR (one of the sixteen genes retrieved as putative miR-146b targets independently predicted by seven miRNA-target prediction software) is affected by miR-146b over-expression. $\mathrm{HuR}$, a ubiquitously expressed member of the HuR family of RNA-binding proteins, has a known role in mRNA stabilization and has been reported to be associated to the ARE-containing 3'UTR of FasL mRNA, which is mandatory for its expression. ${ }^{29,36}$ Remarkably, HuR has been experimentally validated as miR-146b target genes in glioma stem cells ${ }^{31}$ and endothelial cells, ${ }^{30}$ and the predicted miR$146 \mathrm{~b}$ seed region located in the 3'UTR of HuR mRNA has been demonstrated to be functional. ${ }^{30,31}$ According to these published data, restoration of miR-146b in CD8 T-LGLs decreases the levels of HuR mRNA and, consistently, of
FasL. Noticeably, a statistically significant difference in the levels of endogenous HuR protein between CD8 and CD4 T-LGLs is detectable. The suggested pathogenetic link between constitutively active STAT3, defective miR-146b expression and neutropenia, that specifically characterizes the CD8 subset of LGLL, is schematically shown in Figure 7. In conclusion, even though the lack of miR-146b expression may have additional effects on the development and progression of CD8 T-LGL leukemia, in this study we convincingly demonstrated that a STAT3-dependent abrogation of miR-146b expression plays a direct role in the development of neutropenia reported in a subset of LGLL patients, representing a potential target for individualized therapeutic approach.

\section{Funding}

The authors have been supported by AIRC (Milan), by Università degli Studi di Padova, Progetti di Ateneo, by Fondo Unico per la Ricerca (BAZZONI-FUR) and by Fondazione CARIPLO (2015-0584).

\section{References}

1. Lamy T, Moignet A, Loughran TP, Jr. LGL leukemia: from pathogenesis to treatment. Blood. 2017;129(9):1082-1094

2. Semenzato G, Zambello R, Starkebaum G, Oshimi K, Loughran TP, Jr. The lymphoproliferative disease of granular lymphocytes: updated criteria for diagnosis. Blood. 1997;89(1):256-260.
3. Leblanc F, Zhang D, Liu X, Loughran TP. Large granular lymphocyte leukemia: from dysregulated pathways to therapeutic targets. Future Oncol. 2012;8(7):787-801

4. Epling-Burnette PK, Zhong B, Bai F, et al. Cooperative regulation of $\mathrm{Mcl}-1$ by Janus kinase/stat and phosphatidylinositol 3kinase contribute to granulocytemacrophage colony-stimulating factordelayed apoptosis in human neutrophils. J Immunol. 2001;166(12):7486-7495.
5. Teramo A, Barila G, Calabretto G, et al. STAT3 mutation impacts biological and clinical features of T-LGL leukemia. Oncotarget. 2017;8(37):61876-61889.

6. Jerez A, Clemente MJ, Makishima H, et al. STAT3 mutations unify the pathogenesis of chronic lymphoproliferative disorders of NK cells and T-cell large granular lymphocyte leukemia. Blood. 2012;120(15):3048-3057.

7. Koskela HL, Eldfors S, Ellonen P, et al. Somatic STAT3 mutations in large granular 
lymphocytic leukemia. N Engl J Med. 2012; 366(20):1905-1913

8. Oiu ZY, Fan L, Wang L, et al. STAT3 mutations are frequent in T-cell large granular lymphocytic leukemia with pure red cell aplasia. J Hematol Oncol. 2013;6:82.

9. Lamy T, Liu JH, Landowski TH, Dalton WS, Loughran TP, Jr. Dysregulation of CD95/CD95 ligand-apoptotic pathway in $\mathrm{CD} 3(+)$ large granular lymphocyte leukemia. Blood. 1998;92(12):4771-4777.

10. Liu JH, Wei S, Lamy T, et al. Chronic neutropenia mediated by fas ligand. Blood. 2000;95(10):3219-3222

11. Perzova R, Loughran TP, Jr. Constitutive expression of Fas ligand in large granular lymphocyte leukaemia. Br J Haematol. 1997; 97(1):123-126.

12. Tanaka M, Suda T, Haze K, et al. Fas ligand in human serum. Nat Med. 1996;2(3):317322.

13. Ikeda S, Tagawa H. Dysregulation of microRNAs and their association in the pathogenesis of T-cell lymphoma/leukemias. Int J Hematol. 2014; 99(5):542-552.

14. Lawrie CH. MicroRNAs in hematological malignancies. Blood Rev. 2013;27(3):143154.

15. Gattazzo C, Teramo A, Passeri F, et al. Detection of monoclonal $\mathrm{T}$ populations in patients with KIR-restricted chronic lymphoproliferative disorder of NK cells. Haematologica. 2014;99(12):1826-1833.

16. Teramo A, Gattazzo C, Passeri F, et al. Intrinsic and extrinsic mechanisms contribute to maintain the JAK/STAT pathway aberrantly activated in T-type large granular lymphocyte leukemia. Blood. 2013;121(19): 3843-3854, S1.

17. Bazzoni F, Rossato M, Fabbri $M$, et al. Induction and regulatory function of miR-9 in human monocytes and neutrophils exposed to proinflammatory signals. Proc Natl Acad Sci U S A. 2009;106(13):52825287

18. Rossato M, Cencig S, Gasperini S, Cassatella
MA, Bazzoni F. IL-10 modulates cytokine gene transcription by protein synthesis-independent and dependent mechanisms in lipopolysaccharide-treated neutrophils. Eur J Immunol. 2007;37(11):3176-3189.

19. Mohn F, Weber M, Schubeler D, Roloff TC. Methylated DNA immunoprecipitation (MeDIP). Methods Mol Biol. 2009;507:5564.

20. Yu H, Pardoll D, Jove R. STATs in cance inflammation and immunity: a leading role for STAT3. Nat Rev Cancer. 2009;9(11):798809.

21. Lee H, Deng J, Xin H, Liu Y, Pardoll D, Yu H A requirement of STAT3 DNA binding precludes Th-1 immunostimulatory gene expression by NF-kappaB in tumors. Cancer Res. 2011;71(11):3772-3780.

22. Niu G, Wright KL, Ma Y, et al. Role of Stat3 in regulating p53 expression and function. Mol Cell Biol. 2005;25(17):7432-7440.

23. Zhang $\mathrm{O}$, Wang HY, Marzec M, Raghunath PN, Nagasawa T, Wasik MA. STAT3- and DNA methyltransferase 1-mediated epigenetic silencing of SHP-1 tyrosine phosphatase tumor suppressor gene in malignant T lymphocytes. Proc Natl Acad Sci U S A. 2005;102(19):6948-6953.

24. Curtale G, Mirolo M, Renzi TA, Rossato M, Bazzoni F, Locati M. Negative regulation of Toll-like receptor 4 signaling by IL-10dependent microRNA-146b. Proc Natl Acad Sci U S A. 2013;110(28):11499-11504.

25. Renzi TA, Rubino M, Gornati L, Garlanda C Locati M, Curtale G. MiR-146b Mediates Endotoxin Tolerance in Human Phagocytes. Mediators Inflamm. 2015;2015:145305.

26. Villela D, Ramalho RF, Silva AR, et al. Differential DNA Methylation of MicroRNA Genes in Temporal Cortex from Alzheimer's Disease Individuals. Neural Plast. 2016;2016:2584940

27. Xiang M, Birkbak NJ, Vafaizadeh V, et al. STAT3 induction of miR-146b forms a feedback loop to inhibit the NF-kappaB to IL-6 signaling axis and STAT3-driven cancer phenotypes. Sci Signal. 2014;7(310):ra11.
28. Saitoh T, Karasawa M, Sakuraya M et al. Improvement of extrathymic $\mathrm{T}$ cell type of large granular lymphocyte (LGL) leukemia by cyclosporin A: the serum level of Fas ligand is a marker of LGL leukemia activity. Eur J Haematol. 2000;65(4):272-275.

29. Drury GL, Di Marco S, Dormoy-Raclet V, Desbarats J, Gallouzi IE. FasL expression in activated T lymphocytes involves HuRmediated stabilization. J Biol Chem. 2010 285(41):31130-31138

30. Cheng HS, Sivachandran N, Lau A, et al MicroRNA-146 represses endothelial activation by inhibiting pro-inflammatory pathways. EMBO Mol Med. 2013;5(7):1017 1034

31. Yang W, Yu H, Shen Y, Liu Y, Yang Z, Sun T. MiR-146b-5p overexpression attenuates stemness and radioresistance of glioma stem cells by targeting HuR/lincRNA-p21/betacatenin pathway. Oncotarget. 2016;7(27) 41505-41526.

32. Clemente MJ, Przychodzen B, Jerez A, et al. Deep sequencing of the T-cell receptor repertoire in CD8+ T-large granular lymphocyte leukemia identifies signature landscapes. Blood. 2013;122(25):4077-4085.

33. Zhang Q, Wang HY, Woetmann A Raghunath PN, Odum N, Wasik MA. STAT3 induces transcription of the DNA methyltransferase 1 gene (DNMT1) in malignant T lymphocytes. Blood. 2006;108(3):1058-1064

34. Mishra A, Liu S, Sams GH, et al. Aberrant overexpression of IL-15 initiates large granular lymphocyte leukemia through chromosomal instability and DNA hypermethylation. Cancer Cell. 2012;22(5):645-655.

35. Gattazzo C, Teramo A, Miorin M, et al. Lack of expression of inhibitory KIR3DL1 receptor in patients with natural killer cell-type lymphoproliferative disease of granular lymphocytes. Haematologica. 2010;95(10):1722 1729 .

36. Zhu H, Berkova Z, Mathur R, et al. HuR Suppresses Fas Expression and correlates with patient outcome in liver cancer. Mo Cancer Res. 2015;13(5):809-818. 